\title{
Study on Performance and Scheduling Strategy for Lift-sliding Solid Garage based on Queuing Theory
}

\author{
Min Zhang, Shidong Fan \\ School of Energy and Power Engineering, \\ Wuhan University of Technology, \\ Wuhan, 430063, China
}

\begin{abstract}
With the continuous development of urbanization, solid garage is playing a more and more important role in city infrastructure. In this paper, a mathematic model on queuing theory for lift-sliding solid garage is constructed, and some important performance parameters are analyzed base on the quening model. To achieve an optimized operating strategy, three service modes and statistic data are introduced and analyzed, then it reaches the conclusion that different service modes cooperate to realize a optimized working efficiency during different timespans. All the analysis and conclusion are significant for solid garage design and also improving the service performance of the garage by theoretic evidence.
\end{abstract} theory

Keywords-solid garage; scheduling stratety; queuing

\section{INTRODUCTION}

As the urbanization rate is increasing, meanwhile urban per capita area is continuously shrinking, however, the population density and amount of automobiles keep increasing, so solid garage is being a major parking facility especially in dense residential and public spaces in the future. Among different types of solid garage, liftsliding one is popular and accounts for over $80 \%$ in newly-built mechanical garages in emerging metropolis in emerging country like China for its less construction cost, more configuration flexibility, scalability and space adaptability. As auto-parking equipment technology has become more sophisticated, researchers' focus has been gradually shifting from simple hardware configuration to deep discussion and analysis of its operation performance in order to achieve greater efficiency, and better serve people's application.[1] So far there are only limited studies on working efficiency for lift-sliding solid garage. In this context, this paper constructs a mathematical model for solid garage based on queuing theory, achieves some important parameters on the model, and optimizes the accessing strategy by theory and statistical method, which is significant for designing a solid garage with high performance and improving working efficiency of the operating ones.

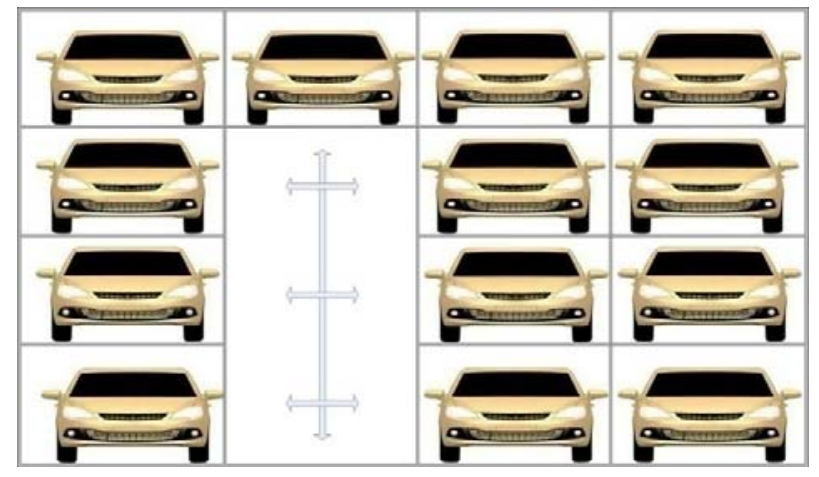

Figure 1. Front view of typical lift-sliding garage

\section{PARKING QUEUE AND QUEUING THEORY}

Queuing theory, also known as stochastic queuing service system theory or waiting queue theory, has main contents of various states probabilities during queuing process, applies to all service systems, and has been widely used in computer networks, manufacturing process, transportation system, inventory management and other stochastic service system of resource sharing. There are three main research fields in Queuing theory: establishing model based on data for statistical inference, probability rules of quantity parameters related to queuing, and system optimization problems. Its purpose is to properly design and effectively operate the service system, and to achieve the best benefits for the system.

Lift-sliding Solid garage is a typical stochastic discrete queue with the automobile as served object and solid garage as service provider.[2,3] So some analytical methods and conclusions in queuing theory are applicable to the parking queue model.

\section{CONSTRUCTING QUEUING MODEL FOR SOLID GARAGE AND PERFORMANCE ANALYSIS}

\section{A. Constructing Queuing Model for Solid Garage}

According to the principles of queuing theory, automobile arrival time follows passion distribution, and the length of service time follows a negative exponential distribution. When parking units reach its maximum $n$, the arriving automobiles will enter the waiting line, theoretically queue length can be assumed to be infinite. Therefore, lift-sliding garage is a multi-channel waiting queue model with discipline of $M / M / n / \infty /$ FCFS 
where the service system is equipped with $\mathrm{n}$ waiters, the parking units. When the service object automobile, arrives, it can be parked with service time following negative exponential distribution if free parking unit is available in the system, otherwise it enters the waiting line, the service discipline is first-come, first-served.

Arrival rate of automobile can be assumed to be $\lambda$, and mean parking time for each automobile is $t$, so average parking rate $\mu=1 / \mathrm{t}$. If the garage's capacity is $\mathrm{n}$, state-transition diagram for the garage is shown as Figure 2 , and state $\mathrm{S}_{\mathrm{k}}$ means the system has $\mathrm{k}$ automobiles including the parked and the waiting ones.[4]

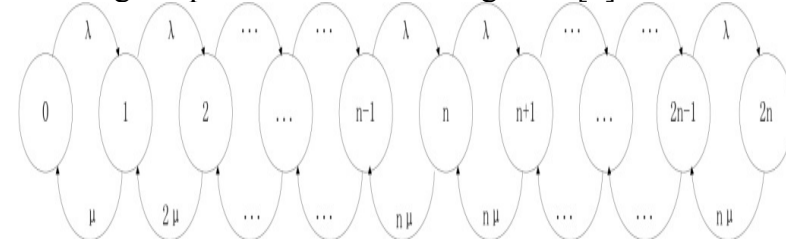

Figure 2. State transition diagram for solid garage

\section{B. Performance Analysis of Parking Queue Model}

Parking Queue is a birth and death chain according to queuing theory. Considering the patient limitation of the drivers, the maximum queue length can be defined $2 \mathrm{n}$, double of parking units, it means when the queue length reaches $2 n$ the new arriving automobiles will not wait in the queue and will be rejected by the system. Automobile arrival rate $\lambda_{i}=\lambda$, parking serving rate

$$
\mu_{i}=i \mu, 1 \leq \mathrm{i} \leq \mathrm{n} ; \mu_{i}=n \mu, \mathrm{n}+1 \leq \mathrm{i} \leq 2 \mathrm{n} . \quad \text { When }
$$

$\lambda<n \mu$, the chain is normal return, and state number is limited, so responding limitation probabilities do exist. According to the system equilibrium theory, the equation of state can be listed as following eqn (1), eqn(2) and eqn (3):

$$
\begin{aligned}
& \text { For } S_{0}, \lambda P_{0}=\mu P_{1} \\
& \qquad \text { For } S_{1} \text { to } S_{n-1}, \\
& (i \mu+\lambda) \mathrm{P}_{i}=\lambda P_{i-1}+i \mu P_{i+1} \quad 0<\mathrm{i}<\mathrm{n}
\end{aligned}
$$

$$
\text { For } S_{n} \text { to } S_{2 n-1} \text {, }
$$$$
(\mathrm{n} \mu+\lambda) \mathrm{P}_{i}=\lambda P_{i-1}+n \mu P_{i+1} \quad \mathrm{n}<\mathrm{i}<2 \mathrm{n}
$$

For regularity,

$$
\sum_{i=0}^{2 n} P_{i}=1
$$

The eqn(1), eqn(2), eqn(3), eqn(4) can be solved, and the solution is as below eqn(5), eqn(6), and eqn(7).

$$
P_{0}=\frac{1}{1+\sum_{i=1}^{n} \frac{1}{i !} \cdot\left(\frac{\lambda}{\mu}\right)^{i}+\sum_{i=n+1}^{2 n} \frac{1}{n !} \cdot\left(\frac{\lambda}{\mu}\right)^{i} \cdot \frac{1}{n^{i-n}}}
$$

$$
\begin{array}{lr}
P_{i}=\frac{1}{i !} \cdot\left(\frac{\lambda}{\mu}\right)^{i} P_{0} & 0<\mathrm{i}<\mathrm{n}+1 \\
P_{i}=\frac{1}{\mathrm{n} !} \cdot\left(\frac{\lambda}{\mu}\right)^{i} \cdot \frac{1}{n^{i-n}} P_{0} & \mathrm{n}<\mathrm{i}<2 \mathrm{n}+1
\end{array}
$$

Substitute eqn(5) into eqn(6) and eqn(7), the limitation probability for the system can be achieved. When the queue length is longer than $\mathrm{n}$, the arriving automobiles have to wait. And the probability of definite waiting for new arrival automobiles is as below eqn(8).

$$
P_{W}=\sum_{i=n+1}^{2 n} P_{i}=P_{0} \cdot \sum_{i=n+1}^{2 n} \frac{1}{n !} \cdot\left(\frac{\lambda}{\mu}\right)^{i} \cdot \frac{1}{n^{i-n}}
$$

The probability of direct parking without any waiting $\mathrm{P}$ is achieved in eqn(9),

$$
P=1-P_{W}=1-\sum_{i=n+1}^{2 n} \frac{1}{\mathrm{n} !} \cdot\left(\frac{\lambda}{\mu}\right)^{i} \cdot \frac{1}{n^{i-n}}
$$

Parking service capability $\mathrm{N}$ in unit time is as below eqn(10),

$$
N=\lambda P=\lambda\left[1-\sum_{i=n+1}^{2 n} \frac{1}{n !} \cdot\left(\frac{\lambda}{\mu}\right)^{i} \cdot \frac{1}{n^{i-n}}\right]
$$

The average utilization rate for parking unit is as eqn(11),

$$
\beta=\frac{\sum_{i=1}^{n} i P_{i}}{n}=\frac{\sum_{i=1}^{n} i \frac{1}{i !} \cdot\left(\frac{\lambda}{\mu}\right)^{i} P_{0}}{n}=\frac{\lambda}{n \mu}\left[1-\frac{P_{0}}{n !}\left(\frac{\lambda}{\mu}\right)^{n}\right]
$$

The service performance of garage is significant to designing a reasonable and efficient garage, and $\mathrm{n}$ is decided by service requirement, $\lambda$ is automobile arrival rate, so in order to fulfill less service rejection rate and increase parking unit utilization rate, some strategies should taken to control average servicing time $\mu$. Some commercial places increase charge for unit time or adopt step-up calculation charging rate to shorten serving time. But for garage in residential area, parking capacity $n$ should be carefully considered when designing because it's not convenient to control the residents' parking time.

\section{ACCESSING STRATEGy OPTIMIZATION FOR LIFT- SLIDING SOLID GARAGE}

Generally, planning and designing solid garage should follow some principles, (1)Service rejection rate is as low as possible, better less than $20 \%$. (2)Waiting queue length and waiting time should not be too long and exceed user's psychological limitation. Nearly $80 \%$ users will abandon parking if waiting time is over 20 minutes. (3) Accessing efficiency is high, which can ensure quick access especially in rush hour and benefit promotion of solid garage technology.[5]

For most widely-used lift-sliding solid garage, the system is composed by a number of independent parking 
subsystems, with total parking units is sum of the independent . Suppose there are $\mathrm{m}$ independent parking subsystems with $n_{j}$ as capacity of subsystem $\mathrm{j}$, the total capacity can be calculated as below eqn(12).

$n=\sum_{j=1}^{m} n_{j} \cdot$

Considering energy saving and accessing efficiency, subsystem should be optimized to be composed by 3 to 10 columns and 2 to 4 floors.

There are two basic accessing strategies for solid garage, one is traditional strategy of First Come First Serve(FCFS), which requires the system to access automobile according to time sequence of accessing order, the other is an optimized strategy, that aims to achieve or approach the least waiting time for the customer, the highest working efficiency and the lowest energy consumption for the garage by ranking the different aims, setting desired values for each aim and calculating the desired optimized value in order before desicion-making.

As for the automobile loader's parking position, there a three methods: (1) Priority of parking or withdrawing. The loader parks at the ground floor waiting for next parking after accessing. (2) Return to original place. The loader goes to its original position after parking and to the ground floor after withdrawing. (3) Interleaving accessing. The automobiles to be parked or withdrawed will be seperated in to two groups, the parking group and the withdrawing one. The system will park and withdraw in turn between two groups. The loader's parking strategy will follow that of return to original place. [6,7]

Since the automobile arrival time and serving time can be assumed to follow passion distribution, the relative data can be simulated. In order to compare different loader parking strategies, simulation data on the accessing time is shown in Figure 3.

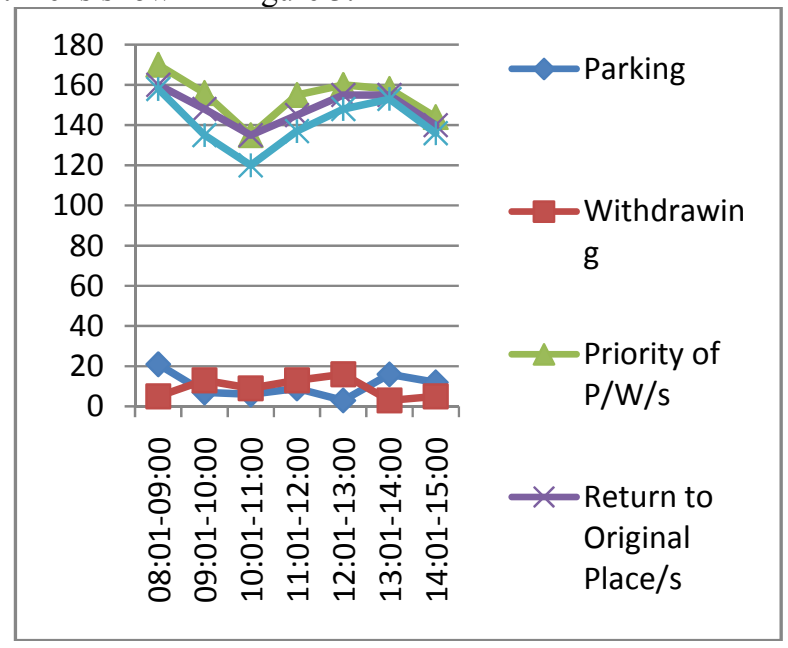

Figure 3. Working Efficiency Comprison of Different Timespans

Conclusion can be drawn from figure 3. Strategy of Return to Original Place can save $3.3 \%$ average operation time comparing with that of Parking/Withdrawing Priority.
Strategy of Interleaving Accessing can take $8.7 \%$ average operation time less than that of Parking/Withdrawing Priority. Working efficiency of Parking/Withdrawing Priority is the lowest particularly while continuous parking task is waiting. And the closer the parking number is to the withdrawing numbers, the more efficiently it works.

\section{CONCLUSION}

Lift-sliding solid garage is an effective solution for parking challenge especially in modern cities with rising population. In this paper, queuing theory is introduced to parking model to analyze performance of lift-sliding solid garage. In addition, studies on operation strategy of the system is also carried on. Different loader parking strategies comparison shows that strategy depends on specific timespan and task characteristic. In the future, we plan to study the relationship between the subsystem configuration type and system performance.

\section{REFERENCES}

[1] Zhou Qicai, Miao Ning,Xiong Xiaolei, Parking Model Setup and Analysis based on Queuing Thoery, Chinese Journal of Cosntruction Machinery, 4, pp.161- 164, 2005

[2] Zhang Fangfang, Liang Fei, Zhu Minze, Research on Lift-sliding steroescopic Garage Control Strategy based on Queuing Thoery, Computer Simulation, 1, pp. 209,2013

[3] S M Tabon, A Simulator for an Automated Warehousing System, Computer and Industrial Engineering, 24(02), pp.281-290, 1993

[4] Lu Chai Lai, Queuing Theory, Press of Beijing University of Post and Telecommunications, 1994

[5] Zhou Xuesong, Tian Mi, Ma Youjie, Ma Yunbin, Shao Baofu, Wang Hui, Research on Optimal Storage-retrieval Control Strategy of the Intelligent Solid Garage, Mamufacturing Automation, 10, pp.32-33, 2008

[6] Hua Wenhan, Tian Huanwen, Research on Storge-retrival Operation Strategy and Optimization of Automatic Steroscopic Garage, Mechanical Design and Mamufacture, 3, pp. 261- 262, 2011

[7] Wang Sufen, The Up-down and Translation Steroe Garage Control Strategy based on Queuing Thoery, Drive System Technique, 4, pp.34-37, 2012 